\title{
Non solo seggioline e tavolini: il valore sociale della proposta di Maria Montessori
}

\author{
Gabriella Seveso* \\ Università degli Studi di Milano-Bicocca
}

Riassunto Il contributo ha un taglio storico con attenzione all'attualità e si occupa della pedagogia montessoriana, che è recentemente al centro di un dibattito vivace in Italia e di nuove sperimentazioni. Esso vuole mostrare il notevole valore etico e sociale presente nella proposta di Maria Montessori: un valore che attualmente è un po' trascurato a livello divulgativo, perché la concezione montessoriana viene ridotta in alcuni casi a un metodo didattico (arredi a misura di bambino, materiali scientifici, maestra unica). Per questo motivo, l'autrice del contributo analizza il Discorso inaugurale in occasione dell'apertura di una Casa dei bambini nel 1907, scritto e pronunciato da Maria Montessori, focalizzandosi su alcuni temi. Il primo tema è quello degli spazi: il Discorso mette in luce come Montessori pensava al valore simbolico e sociale delle Case dei bambini e alla loro importanza per il recupero di situazioni di degrado e di miseria. Il secondo tema è quello del profilo professionale della maestra: le parole di Montessori mostrano come l'insegnante ha una importante funzione sociale ed è la persona che cresce i futuri cittadini favorendone la consapevolezza critica attraverso una relazione fondata sull'autonomia. Una rilettura dell'opera montessoriana in questa luce ci sprona anche a rivedere le proposte di altri pensatori della nostra storia culturale non come metodi didattici, pur innovativi e interessanti, ma anche e soprattutto nel loro messaggio sociale. Questa prospettiva appare sempre più urgente e doverosa in un'epoca come la nostra, che si trova ad affrontare problemi molto gravi riguardo alle condizioni dell'infanzia.

PAROLE CHIAVE: Valore sociale della pedagogia montessoriana; Progettazione degli spazi montessoriani; Ruolo dell'insegnante montessoriana. 
Not just chairs and tables: the social value of the proposal by Maria Montessori

Abstract The contribution has a historical focus with attention to current affairs and deals with Montessori education, which has recently been at the centre of a lively debate in Italy and new experiments. It wants to show the remarkable ethical and social value present in the proposal of Maria Montessori: a value that is currently somewhat neglected at the level of popularization because the Montessori concept is reduced in some cases to an educational method (child-friendly furnishings, materials and methods, single teacher). For this reason, the author of the contribution analyzes the inaugural speech on the occasion of the opening of a Children's Home in 1907, written and pronounced by Maria Montessori, focusing on some themes. The first theme is that of spaces: the Discourse highlights how Montessori thought of the symbolic and social value of the homes of children and their importance for the recovery of situations of degradation and misery. The second theme is that of the professional profile of the teacher: the words of Montessori show how the teacher has an important social function and is the person who grows future citizens, encouraging critical awareness through a relationship based on autonomy. A reinterpretation of the Montessori work in this light also encourages us to review the proposals of other authors of our cultural history not as educational methods, although innovative and interesting, but also and above all in their social message. This perspective appears ever more urgent and necessary in our age, which is facing very serious problems concerning the conditions of childhood.

KEYWORDS: The social value of Montessori pedagogy; Design of Montessori spaces; Role of the Montessori teacher.

\section{Não apenas cadeiras e mesas: o valor social da proposta de Maria Montessori}

Resumo Este artigo tem uma abordagem histórica com a atenção voltada a atualidade e se ocupa da pedagogia montessoriana, que recentemente esteve no centro de um vivaz debate na Itália e novas experimentações. $\mathrm{O}$ presente artigo quer mostrar o notável valor ético e social presente na proposta de Maria Montessori: um valor que atualmente é um pouco negligenciado quando da sua divulgação, porque a concepção montessoriana é reduzida em alguns casos a um método didatico (móveis nas medidas das crianças, materiais científico, professora única). Por este motivo, a autora analisa o Discurso inaugural por ocasião da abertura de uma "Casa dei Bambini" em 1907, escrito e pronunciado por Maria Montessori, destacando alguns temas. O primeiro tema é o dos espaços: o Discurso foca em como Montessori pensava o valor simbólico e social 
das "Casas dei bambini" e sua importância para a recuperação de situações de degradação e miséria. O segundo tema é o do perfil profissional da professora: as palavras de Montessori mostram como a professora tem uma função social importante e é a pessoa que cultiva os futuros cidadãos, favorecendo a consciência crítica por meio de uma relação baseada na autonomia. Uma releitura da obra montessoriana deste ponto de vista também nos encoraja a rever as propostas de outros pensadores de nossa história cultural não como métodos didáticos, embora inovadores e interessantes, mas também e sobretudo em sua mensagem social. Essa perspectiva parece cada vez mais urgente e necessária em uma época como a nossa, que enfrenta problemas muito sérios com relação às condições da infância.

PALAVRAS-CHAVE: Valor social da pedagogia montessoriana; Design de espaços Montessori; Papel do professor Montessori.

\section{Introduzione}

" Anche a Bicêtre, ove mi trattenni lungamente a studiare, vidi che si adottavano più i meccanismi didattici che il sistema del Séguin; tuttavia il testo francese era in mano agli educatori»: così scrive Maria Montessori (1999b, p. 27) nelle prime pagine del suo libro La scoperta del bambino ${ }^{1}$ dedicate alla storia dei metodi: l'autrice riconosce come pietre miliari nella storia della pedagogia l'opera di J. Itard e quella di É. Séguin, ma ricorda, a proposito del suo lungo soggiorno di studio in Francia, come il metodo di quest'ultimo fosse ormai riprodotto in maniera stereotipata e meccanica. Queste riflessioni la portano, quindi, a mettere in guardia dal pericolo di ridurre un metodo a un insieme di pratiche e di materiali didattici perdendo di vista lo spessore culturale e la più complessa cornice di riferimento di una proposta pedagogica. Si tratta di considerazioni che risultano molto attuali, in Italia, se pensiamo al fatto che recentemente il pensiero e l'opera di autori o autrici che appartengono alla nostra storia culturale viene riproposto come metodo didattico, ma dimenticando il messaggio sociale che esso aveva originariamente. Per esempio, in alcuni casi, l'opera di Freinet è ridotta e conosciuta nelle scuole come metodo che propone un approccio naturale all'apprendimento della scrittura, un uso del testo libero, un'attenzione al lavoro di gruppo: in questa riduzione, a volte presente in alcune istituzioni scolastiche italiane, è dimenticata o sottovalutata la portata sociale del pensiero di Freinet, con tutte le sue riflessioni sui mutamenti della società, sulle funzioni della scuola, sul problema di formare futuri cittadini dotati di spirito critico e di consapevolezza in un mondo in cui va affermandosi sempre più un sistema economico che crea fragilità e marginalità. Lo stesso pericolo a volte si presenta riguardo all'opera di Montessori, che risulta in alcuni casi conosciuta dagli insegnanti più per gli aspetti di metodo che per le considerazioni sociali originarie.

Leggendo la parte introduttiva che abbiamo citato all'inizio di questo contributo, è possibile rendersi conto di come invece il metodo che Maria Montessori mette a punto gradualmente è frutto di una lunga speculazione dettata da molteplici suggestioni e di come la pedagogia sperimentale e scientifica che intende fondare non 
è meramente un esperimento fattuale, ma si basa su un'analisi e su considerazioni epistemologiche molto complesse e articolate e si colloca su uno sfondo di profonda tensione etica e sociale (TOGNON, 2016). Per questo motivo, l'opera della Montessori non può essere ridotta, anch'essa, a quegli elementi più vistosi e conosciuti, più apertamente dirompenti, delle pratiche didattiche (gli arredi a misura di bambino, l'importanza degli spazi, la maestra unica ecc.), come a volte attualmente accade a livello divulgativo, nel nostro Paese: essa va conosciuta, come hanno più volte sottolineato alcuni studiosi e ricercatori, in tutta la sua complessità, nella sua ricchezza anche speculativa, nel suo spessore teorico ed etico.

All'interno di questo nostro contributo, a partire dalla lettura di un'opera molto celebre quale il Discorso inaugurale in occasione dell'apertura di una Casa dei bambini nel 1907, intendiamo dunque riflettere su alcuni particolari aspetti della proposta montessoriana, mettendone in luce la forte rilevanza sociale.

Pur non essendo fra gli scopi del presente lavoro, vorremmo molto sinteticamente rammentare alcuni elementi di cornice: innanzitutto il periodo che abbraccia la fine del XIX secolo e l'inizio del XX secolo, per quanto riguarda la storia delle istituzioni scolastiche ed educative, rappresenta un momento di particolare pregnanza (BECCHI, 1996), poiché, a seguito della raggiunta Unità d'Italia, la scuola diviene oggetto di interventi politici numerosi e considerevoli, anche se non sempre coerenti ed efficaci, proprio perché il problema di educare il nuovo popolo italiano creando anche una coscienza nazionale, si poneva come centrale nel dibattito del tempo, così come la diatriba sulla necessità o meno di educare le masse o semplicemente le élites, diatriba accesasi già vivacemente fin dall'inizio dell'Ottocento, era ancora molto intensa e sentita (MACINAI, 2006; MERLO, 2011). A livello di provvedimenti legislativi, ci sembra opportuno ricordare come il Regio Decreto n. 5 del 16 febbraio 1888 vide l'istituzione dei patronati scolastici, che però era facoltativa e a carico dei Comuni: questo provvedimento, volto, come il legislatore sottolineava, ad aiutare i ragazzi poveri, di fatto suggeriva la necessità di sostenere l'istruzione delle classi più disagiate, ma al tempo stesso mostrava lo scarso impegno dello Stato nel perseguire questo obiettivo, data la delega agli Enti locali, le cui casse erano dissanguate, e dato il carattere opzionale dell'attuazione della legge (DI POL, 2016).

A fronte di questa scarsa sensibilità, o addirittura opposizione all'istruzione popolare, manifestata dalle incoerenze dei provvedimenti legislativi nazionali, il mondo culturale del tempo mostrava notevole interesse verso il tema della funzione sociale della scuola e delle scienze. È possibile ricordare a questo proposito l'impegno profuso dalle Associazioni femminili per la tutela dei diritti dell'infanzia e per la promozione dell'accesso all'istruzione di bambini e bambine (SEVESO, 2018): questo impegno vide notevoli e numerose iniziative promosse sia da gruppi sia da singole personalità, quali, solo a titolo di esempio, Unione Femminile a Milano (BUTTAFUOCO, 1995; MAPELLI; SEVESO, 2006). Un'ulteriore testimonianza molto significativa di tale interesse può essere rinvenuta sia nella vivacità delle iniziative dell'associazionismo presente in molte centri, sia nei progetti di coordinamento di studiosi e/o di divulgazione avviati in alcune città italiane. A quest'ultimo proposito, basti citare l'ambizioso piano per istituire una rete che coordinasse tutti gli Enti e le istituzioni scientifiche e 
formative, che a Milano vide impegnate numerosissime personalità della cultura, delle scienze e della politica locale: il progetto, che giunse a fondare la rete del Politecnico e quella degli Istituti di Perfezionamento, intendeva di fatto coordinare le discipline, i differenti approcci, i luoghi di ricerca e sperimentazione, le metodologie, con un obiettivo esplicitamente sociale e di ricaduta sulla vita della città e all'interno di un dialogo aperto e vivace con gli esponenti della realtà imprenditoriale (CANADELLI, 2008; ZOCCHI, 2008). ${ }^{2} \mathrm{E}$ curioso ricordare come il progetto vide impegnato in prima fila, con passione e con prese di posizione molto esplicite e mirate, anche Antonio Stoppani, ritenuto lo zio di Maria Montessori, ${ }^{3}$ geologo e divulgatore, attento all'edificazione e alla conduzione di quello che resterà un importantissimo ente milanese, ovvero il Museo Civico di Storia Naturale (LIVI, 2008). Come vedremo oltre, ad un simile progetto può essere accostato il disegno di risanamento di spazi urbani e di recupero sociale che porta, a Roma, alla ristrutturazione del quartiere San Lorenzo e alla realizzazione della Casa dei bambini montessoriana (DE SANCTIS, 2013). Questa forte sensibilità verso la funzione sociale delle scienze e dell'educazione era dunque molto presente nel mondo culturale del tempo ${ }^{4}$ e costituisce una dimensione che rileva in tutta la sua originalità e pregnanza nell'opera di Montessori (CIVES; TRABALZINI, 2017; REGNI, 2007), come appare evidente, fra l'altro, nelle prime opere montessoriane (MONTESSORI, 1898; MONTESSORI, 1904).

\section{La progettazione degli spazi nella sua valenza sociale}

La vigorosa tensione etica e la forte valenza sociale sentita da Maria Montessori appare evidente proprio nel suo Discorso inaugurale in occasione dell'apertura della seconda Casa dei bambini, pronunciato nel 1907 a Roma, che si apre con un incipit molto risoluto:

Può darsi che la vita dei poveri sia una cosa, che qualcuno di voi, qui presente, non abbia mai considerato in tutta la sua degradazione. Può darsi che abbiate sentito la miseria della estrema povertà umana soltanto attraverso le pagine di qualche grande libro, o la vibrante voce di un grande attore (MONTESSORI, 1999a, p. 361).

Così denuncia l'autrice, sottolineando poi come il quartiere San Lorenzo, dove si apre la Casa, non è il quartiere del popolo, è il quartiere dei poveri» (p. 362). A partire da quest'ultima constatazione, il discorso propone una ricostruzione della crisi edilizia e della conseguente speculazione che ha deformato il quartiere stesso sia da un punto di vista architettonico sia da un punto di vista sociale, fino a renderlo un luogo di «agglomeramento, promiscuità, immoralità» (ibidem). L'atteggiamento dell'autrice è lucido, ma non giudicante: Maria Montessori non addita gli abitanti di San Lorenzo come delinquenti da condannare, ma neanche nega la realtà di degrado sociale e morale nella quale essi sono immersi e che quotidianamente vivono e rinnovano. Nella sua concretezza, passa a ricordare dunque le incongruenze di alcuni dibattiti pedagogici, che affrontano tematiche connesse alla didattica senza rendersi conto dei fattori sociali che condizionano la vita degli scolari: ribadisce, infatti come, quando si dibatte se affidare o meno i compiti a casa ai bimbi, non ci si accorge che nelle case di questi bambini non c'è neanche la luce per poter scrivere. L'esempio, molto pragmatico e quotidiano, mostra come Maria Montessori avesse ben chiaro il pericolo di 
una riflessione didattica svincolata dalle considerazioni sui fattori sociali, aspetto che già era rilevato in alcune sue opere molto interessanti di qualche anno precedenti. Si veda, in particolare, la ricerca da lei condotta sull'influenza delle condizioni familiari sul livello intellettuale degli scolari (MONTESSORI, 1904), all'interno della quale l'autrice mostrava una correlazione fra provenienza sociale e culturale e rendimento scolastico e denunciava il ruolo repressivo dell'istituzione scolastica nei confronti dei più svantaggiati: l'autrice sostiene infatti che «la scuola contribuisce soltanto ad aggravare condizioni sociali ingiuste, castigando nel bambino la miseria, la malattia, la sventura» (PIRONI, 2010d, p. 55).

A partire da queste considerazioni, Maria Montessori nel Discorso inaugurale illustra ai suoi ascoltatori l'importanza della progettazione dello spazio e perfino dei termini utilizzati per designarlo:

\begin{abstract}
Se pensiamo all'idea dogmatica e poetica che ci siamo fatti della casa, elevata sino al significato quasi sacro della 'home' inglese, il tempio chiuso dell'intimità inaccessibile a chi non è caro, e se riflettiamo al gran contrasto e alla crudeltà di infondere come sentimento educativo questo della casa, in tutti - mentre tanti non hanno casa, ma soltanto mura luride $[\ldots]$ - dobbiamo allora concludere che non possiamo parlare in astratto di casa, e farne motivo generico di educazione delle masse, e parlarne come del fondamento che dà, con la famiglia, saldezza alla compagine sociale (MONTESSORI, 1999a, p.363).
\end{abstract}

Sembra evidente una riflessione molto profonda, accorata, preoccupata, sui problemi sociali e un atteggiamento di denuncia dello sfaldamento della società; per i poveri, conclude l'autrice, è più intima, più decorosa e igienica la strada. L'analisi del termine "casa" dà il via, inoltre, ad una disamina molto articolata su quanto, nel corso dei secoli, le case di ricchi e dei meno abbienti fossero vicine e permettessero una sorta di scambio fra classi sociali, mentre nella realtà contemporanea alla Montessori si era ormai prodotto - sottolinea l'autrice - un processo di isolamento dei poveri, lasciati a se stessi, relegati in alcuni quartieri, allontanati dal contatto con classi sociali più elevate:

Siamo venuti così cercando dei focolari infetti, che dovrebbero significare pericolo e minaccia, per chi ha coscienza sociale, per la città che è depurata all'interno da tutto ciò che è brutto, e che si è ammalata di cancrena, volendo farsi tutta bella e linda, perseguendo un aristocratico ideale estetico. (MONTESSORI, 1999 a, p. 364).

È evidente come il termine "casa" non sia utilizzato dalla Montessori semplicemente per segnalare la serenità di un luogo intimo, per indicare le soluzioni di arredamento e di design simili alla domesticità (le seggioline, i tavolini), come a volte attualmente, soprattutto a livello divulgativo, si intende pensando alla proposta montessoriana. L'autrice usa il termine "casa" insistendo sul significato simbolico e sociale e per denunciare le condizioni di degrado e la necessità di risolvere tali condizioni: la Casa dei bambini vuol essere un modo per affrontare questo degrado e soprattutto questo processo di allontanamento, di esclusione, di repressione (PIRONI, 2010 a; PIRONI, 2010c). Non a caso, la Montessori prosegue il suo discorso raccontando con 
veemenza come, attraversando per la prima volta il quartiere, abbia avuto la sensazione di un disastro e di un lutto, quasi come se si potessero interpretare le tracce prima di un'inondazione, poi di un terremoto. D'altro canto, occorre ribadire come il progetto all'interno del quale si colloca la Casa dei bambini di San Lorenzo, è un'iniziativa particolare, promossa dall'Istituto dei Beni Stabili del Comune di Roma, e coordinato dall'ingegner Talamo: le case fatiscenti sono infatti acquistate e ristrutturate dal Comune come abitazioni più contenute e più moderne, ottenendo di offrire ad ogni famiglia un'intimità, uno spazio privato; la manutenzione degli stabili resta però a carico degli inquilini, che vengono in questo modo responsabilizzati; all'interno di uno questi edifici, nella sala al piano terra, è collocata la Casa dei bambini, con l'obiettivo di offrire riparo ai bambini in età prescolare: anche la manutenzione di quest'ultima richiede la partecipazione degli abitanti del quartiere. È dunque evidente che la proposta montessoriana si inserisce in un progetto che mira non solo al recupero urbanistico ed architettonico, ma a mettere in atto un nuovo modo di abitare, e a realizzare spazi che creino responsabilità, senso civico, cultura della partecipazione (SCOCCHERA, 2005). La Casa dei bambini si colloca in queste cornice, (che oggi definiremmo di housing partecipato), di responsabilizzazione e di compartecipazione, ci mostra cioè come la proposta di spazi e arredi educativi nuovi non possa essere sganciata e isolata da ciò che cè al di fuori della scuola: si tratta di un legame profondo fra istituzione educativa e scolastica e territorio; è un modello significativo e incisivo di rivendicare con forza il valore sociale dell'educazione e anche il valore sociale della progettazione architettonica e del design (TORNAR, 2007; TRABALZINI, 2018). Maria Montessori sostiene che in questo contesto non si ha una casa, ma la si sa abitare: il senso della parola abitare acquista in questa cornice un significato profondo ed ampio, non di prendere meramente possesso, ma di continuare a frequentare un luogo per prendersene cura, per giungere alla consapevolezza del suo valore e per sentirsene responsabili.

Il discorso cita anche più volte l'impegno che il Comune richiede agli abitanti del quartiere per prendersi cura degli stabili e della Casa dei bambini non solo in termini di manutenzione e pulizia, ma anche in termini di abbellimento e ornamento: «il popolo acquista così, insieme con il sentimento della casa, quello della pulizia, che fa parte del sentimento estetico, educato anche dagli ornamenti naturali: piante numerose, rampicanti, vasi di fiori nei cortili» (MONTESSORI, 1999 a, p. 364). In questo passaggio emerge con chiarezza uno dei temi fondamentali dell'opera motessoriana, ovvero la progettazione degli spazi intesa come cura e come bellezza, e l'innegabile valore sociale della dimensione estetica, istanza molto presente anche in autrici coeve, prima fra tutte Elle Key, che in quegli anni aveva promosso una concezione non élitaria della fruizione artistica e aveva sottolineato come la bellezza fosse una dimensione fondamentale della promozione sociale. Maria Montessori riprende ed esplicita questa idea in numerosi passaggi dei suoi scritti e puntualizza, ad esempio, l'idea che la bellezza di un interno "non è fatta in questo caso col "superfluo", "col lusso", ma con la grazia e l'armonia delle linee e dei colori, uniti a quella massima semplicità che è richiesta dalla "leggerezza" del mobile» (1999b, p. 127). Ciò che è "bello", dunque, non corrisponde ad un'esibizione di caratteri vistosi o riccamente fascinosi, ma con il decoro, la semplicità, l'armonia, che rimandano e permettono un equilibrio interno, psichico, delle persone, e che richiedono un esercizio di cura che diviene promozione 
sociale: «affermando il valore sociale dell'arte e la sua conseguente valenza pedagogica, - scrive a questo proposito Tiziana Pironi - la fruizione estetica rappresentava un efficace antidoto contro la degradazione e la disgregazione della personalità umana; vivere in un ambiente bello e curato rendeva più felici e migliori» (2010d, p. 70). Nel caso della Casa dei bambini del quartiere San Lorenzo, Maria Montessori ricorda come la valenza pedagogica e di promozione sociale della dimensione estetica siano resi ben evidenti dall'istituzione di un premio erogato da Comune per gli inquilini che meglio si prenderanno cura della loro abitazione: l'autrice definisce questa soluzione una "gara sana e nobilitante», che ha portato già nei primi due anni dall'edificazione ad una maggiore responsabilità sociale e consapevolezza del vivere civile.

\section{Il ruolo delle insegnanti: la maestra e il contesto sociale}

Nel Discorso inaugurale, Maria Montessori si focalizza anche con molta passione e accuratezza sul ruolo della direttrice della Casa:

La direttrice è sempre a disposizione delle madri e la sua vita di persona colta e civile è costante esempio agli abitanti della casa, perché essa ha l'obbligo imprescindibile di alloggiare nel casamento e di essere quindi la coinquilina delle famiglie di tutti i suoi allievi (1999a, p. 367).

Si tratta di un tema molto delicato, che richiama il dibattito molto vivace del tempo sia sul ruolo delle direttrici e delle maestre, sia sulle relazioni fra istituzioni scolastiche e famiglia (MONTESSORI, 2000; PIRONI, 2017; TRABALZINI, 2016). La Montessori si trova ad operare in un particolare momento storico, in cui la partecipazione delle donne alle iniziative educative si fa più intensa, raggiunge una certa solidità sulla scena pubblica, si fa portatrice di numerose istanze sociali: ne sono testimonianza la fondazione di numerose associazioni molto vivaci nei più importanti centri italiani e anche la diffusione di alcune riviste coordinate e scritte da donne (cfr. BUTTAFUOCO, 1995; MAPELLI; SEVESO, 2006). Solo a titolo di esempio, basti pensare, per la realtà milanese, alla fondazione di Unione Nazionale Femminile, ad opera di Ersilia Bronzini Majno, Edvige Vonwiller, Ada Negri, Jole Bersellini ed altre e all'omonima rivista (PIRONI, 2018); o ancora all'opera di alcune donne che oltre ad impegnarsi per la promozione sociale, furono promotrici e sostenitrici proprio del metodo montessoriano: Alice Hallgarten Franchetti, che mise a disposizione la propria tenuta della Montesca a Città di Castello per una Casa dei bambini e un centro di studio; Maria Maraini Guerrieri Gonzaga che aprì a Palidano una Casa dei Bambini (ALATRI, 2018; PIRONI, 2010b); o, nella capitale, Lavinia Taverna e Virginia Nathan, moglie del sindaco di Roma Ernesto Nathan, che favorì la fondazione della prima Casa dei bambini nel 1907. A questo proposito, Antonella Cagnolati sottolinea come molte furono le

figure femminili che, in un periodo quanto mai significativo per la crescita dell'alfabetizzazione e per la diffusione dell'idea stessa di scolarità, operarono nell'ambito dell'istruzione sia come portatrici di nuove teorie pedagogiche, sia sul fronte dell'insegnamento pratico e quotidiano negli istituti scolastici, sia in qualità di giornaliste e scrittrici. (2010, p 11). 
Si tratta di un fenomeno non solo italiano, ma presente in molte zone d'Europa, come sottolinea Hugh Cunningham, calcolando, ad esempio, che solo in Inghilterra, all'inizio del XX secolo, cinquecentomila donne lavoravano «continuativamente e semiprofessionalmente» nella filantropia e partecipavano ad iniziative di promozione sociale destinate all'infanzia (1995, p. 169).

Accanto a questo fenomeno, l'ingresso massiccio delle donne nel mondo della scuola e la conseguente rapida femminilizzazione dell'insegnamento ponevano anche al centro del dibattito politico e culturale il tema della formazione delle maestre e quello della definizione del loro ruolo professionale (COVATO, 1996). Alcuni provvedimenti legislativi testimoniano della complessità e dell'urgenza della problematica: nel 1879 vengono istituite le Scuole magistrali rurali per formare gli insegnanti in due anni; il ministro Coppino, in quegli anni, lavora sul riordino dello stato giuridico e economico degli insegnanti; nel 1896 la riforma Gianturco istituisce uno specifico corso frobeliano per le maestre dei giardini di infanzia (si propone l'insegnamento delle discipline, la pedagogia frobeliana, la raccomandazione ad assumere un atteggiamento materno); nel 1916 sono istituite le Scuole pratiche magistrali per le educatrici di infanzia (biennali) con accesso o dopo tre anni di scuola secondaria o al compimento dei 14 anni. A questo proposito, Di Pol sottolinea come

i primi venti anni del secolo XX registrarono sul riordinamento dell'istruzione magistrale un ampio e anche costruttivo confronto a livello si pedagogico-culturale, sia legislativo che produsse numerose proposte e progetti di riforma, ma che nel concreto non andarono oltre l'attuazione di interventi parziali e di scarsa portata, insufficienti rispetto alle emergenti richieste formative della società italiana. (2016, p. 88).

A fronte di questi importanti e complessi fenomeni, Maria Montessori prende attivamente posizione, delineando nelle sue opere con molta chiarezza il ruolo e il profilo professionale sia delle direttrici sia delle maestre e impegnandosi anche concretamente in alcune iniziative di formazione delle insegnanti, poiché diviene docente di Antropologia presso la scuola pedagogica di Roma e presso l'istituto Superiore di Magistero Femminile. Fin dalla sua partecipazione al Congresso Femminista di Londra, nel 1899, Maria Montessori denuncia le inique condizioni delle insegnanti e nel Primo Congresso Pedagogico Nazionale di Torino affronta il tema del ruolo sociale della scuola e ricorda l'emergenza relativa alla formazione delle maestre in Italia (GALLERANI, 2010; TRABALZINI, 2003); connette comunque queste riflessioni alla necessità di un profondo rinnovamento del modello di scuola, senza il quale non sarebbe possibile anche un'efficace riqualificazione delle insegnanti. Certamente, alcune delle posizioni assunte dall'autrice sono molto vicine a quelle di altre personalità femminili del tempo e di alcune associazioni (BABINI; LAMA, 2000): Linda Malnati, ad esempio, insegnante, direttrice, scrittrice e redattrice di La difesa delle lavoratrici, più volte aveva sottolineato l'esigenza di fornire adeguata preparazione alle future insegnanti, l'urgenza di elevare la preparazione culturale delle maestre, la necessità di sottrarre l'accesso alla professione agli arbitri delle autorità locali (SEVESO, 2018). La proposta montessoriana, però, inserisce questa problematica in una riflessione molto più vasta e molto più profonda sul ruolo della scuola e delle maestre nella società: 
tutto ciò risulta estremamente evidente già dal passo citato ad inizio paragrafo, nel quale Maria Montessori sottolinea come la direttrice della Casa dei bambini risieda all'interno del quartiere, in parte riprendendo alcuni modelli proposti dall'associazionismo inglese, che sosteneva come fosse indispensabile vivere nello stesso ambiente delle persone svantaggiate per consentire loro di mutare la propria condizione. La direttrice e la maestra sono per la Montessori dei modelli di vita, di elevazione culturale di rettitudine morale; a loro l'autrice chiede di essere dedite alle madri e ai bambini ponendosi al di sopra dei pregiudizi proprio per favorire la crescita libera e autonoma di questi ultimi, come appare anche in opere successive: "L'insegnante dovrebbe lavorare al servizio non di un credo politico o sociale, ma dell'essere umano nella sua completezza, in grado di esercitare liberamente, con autodisciplina, la volontà e il giudizio, non sviato da pregiudizi e non distorto da paure" (MONTESSORI, 2007, p. 11). In questo senso, appare chiaro come il ruolo dell'insegnante muta non solo perché riveste nuove funzioni e perché si muove all'interno di una scuola differente, ma anche perché è costantemente ancorato alla sua funzione sociale, di persona che cresce $i$ futuri cittadini favorendone la consapevolezza critica attraverso una relazione fondata sull'autonomia. Scrive l'autrice in La scoperta del bambino: "La maestra diventa prudente, delicata e multiforme. Non abbisognano le sue parole, la sua energia, la sua severità, ma quel che occorre è la sapienza oculata nell'osservare, nel servire, nell'accorrere o nel ritirarsi..." (1999b, p. 165), ma questo nuovo compito a cui l'insegnante è chiamata è finalizzato a promuovere proprio la libertà dell'allievo e a favorirne l'emergere delle risorse: "La maestra non fa altra cosa che aiutarlo in principio ad orientarsi tra tante cose diverse a da apprenderne l'uso preciso, cioè lo inizia alla vita ordinata e attiva nell'ambiente; ma poi lo lascia libero nella scelta e nell'esecuzione del lavoro" (1999 a, p. 69). In questa cornice, risulta chiaro anche il compito primario dell'insegnante e della scuola, di mettersi in relazione di costante e proficua collaborazione con le famiglie, come evidente ancora una volta dalle parole pronunciate nel Discorso inaugurale: "Chi ha pratica della scuola e dei principali problemi pedagogici che la riguardano, sa come venga considerato un grande principio - principio reale e quasi irrealizzabile l'armonia degli intenti educativi fra la famiglia e la scuola. Ma la famiglia è qualche cosa di sempre lontano e di quasi ribelle; una specie di fantasma irraggiungibile per la scuola" (1999a, p. 368). Ora, proprio il fatto che la Casa dei bambini è parte integrante del quartiere ed è percepita dalle famiglie come luogo collettivo, di benessere comune e di comune responsabilità, fa sì che la relazione fra maestra e famiglie sia improntata alla disponibilità e all'aiuto reciproco. La Montessori parla, a questo proposito, di "casa socializzata», all'interno della quale tutte le madri possono godere del privilegio di affidare le cure dei propri bambini alla maestra, così come fino a quel momento solo le donne più agiate potevano fruire delle cura di un'istitutrice per i propri figli; l'autrice giunge a paragonare la "carta biografica" del bambino, da lei introdotta nella Casa dei bambini quale strumento di lavoro e di osservazione, ai "carnet maternel" di cui potevano fruire le nobildonne per annotare misure o principali eventi della vita dei propri figli. Lesito di questa felice relazione fra maestra e famiglie, fra scuola e territorio è materialmente reso evidente dai doni che le madri depongono presso il davanzale della scuola: dolci, fiori, quale «omaggio muto, reverente, quasi religioso» (MONTESSORI, 1999 a, p. 369). La Montessori giunge a definire questo processo una socializzazione della funzione materna, intendendo la dimensione del materno 
come dimensione di cura non solo delle nuove generazioni, ma di tutta la società e il creato, in una concezione molto ampia e universale, che salda le istanze sociali con il sentimento religioso.

\section{Una breve riflessione conclusiva}

Mario Montessori ricorda come la madre, al momento della morte il 6 maggio del 1952, si stava preparando per un viaggio in Ghana, pronta a prestare la sua opera laddove sapeva ci fossero bambini bisognosi. Purtroppo, questa aspetto è a volte attualmente trascurato, soprattutto a livello divulgativo, perché in Italia le scuole a metodo Montessori hanno conosciuto complesse vicissitudini che le hanno portate ad essere private e quindi vengono percepite come scuole accessibili solo a chi ha disponibilità finanziarie.

Il commovente particolare biografico riportato sopra, invece, ci ricorda come la proposta montessoriana fosse sempre tesa ad affrontare i problemi reali della società: essa, infatti, non nasce e si diffonde come semplice metodo didattico, ricco di soluzioni operative interessanti ed innovative, ma come riflessione complessa, articolata, interdisciplinare che sottolinea anche la forte valenza sociale della scuola. Questo aspetto, che ci invita a uno sguardo non riduzionista sulla Montessori, risulta oggi molto attuale, di fronte ad una società che deve affrontare le problematiche connesse a nuove povertà educative e a nuove forme di esclusione: ancora una volta, dunque, sembrano significative ed illuminanti alcune parole presenti in uno scritto dell'autrice, Come educare il potenziale umano:

Di rado un assassino o un ladro è diverso dagli altri bambini al momento della nascita, ma le condizioni in cui cresce gli impediscono di adattarsi alle leggi del suo paese. Le condizioni sociali agiscono sul fisico e sul morale e l'individuo diventa anormale: di solito il criminale riflette gli errori della società. (MONTESSORI, 2007, p. 171).

Una rilettura dell'opera montessoriana in questa luce ci sprona anche a rivedere le proposte di altri pensatori della nostra storia culturale non come metodi didattici, pur innovativi e interessanti, ma anche e soprattutto nel loro messaggio sociale. Questa prospettiva appare sempre più urgente e doverosa in unepoca come la nostra, che si trova ad affrontare problemi molto gravi riguardo alle condizioni dell'infanzia: le disparità sociali e economiche, le migrazioni, la violenza, le differenze di ogni tipo. Di fronte a questi fenomeni così laceranti, leggere le opere di alcuni autori solo come metodi didattici (il lavoro di gruppo, il testo, gli arredi) risulta fuorviante e comodo, perché riduce una proposta pedagogica a tecniche o a soluzioni concrete. Ma il pensiero di alcuni autori e autrici ci ricorda il forte nesso fra scuola, educazione, società e la funzione di cambiamento e di critica che la pedagogia dovrebbe rivestire per costruire una futura società migliore, capace di evitare le marginalità e le fragilità che purtroppo sempre più spesso invece si moltiplicano. 


\section{Referências}

ALATRI, Giovanna. Il mondo al femminile di Maria Montessori. Regine, dame e altre donne. 1. Ed. Roma: Fefè Editore, 2015.

BABINI, Valeria; LAMA, Luisa. Una donna nuova, Il femminismo scientifico di Maria Montessori. 1.Ed. Milano: FrancoAngeli, 2000.

BECCHI, Egle. Il nostro secolo, in BECCHI, Egle; JULIA, Dominique (orgs.). Storia dell'infanzia. Vol. II. Dal Settecento ad oggi, 1 Ed. Roma-Bari: Laterza, 1996. p. 332-406.

BUTTAFUOCO, Annarita. Questioni di cittadinanza. Donne e diritti sociali nell'Italia liberale.1 Ed. Siena: Protagon Editori Toscani, 1995.

CAGNOLATI, Antonella (org.). Maternità militanti. Impegno sociale tra educazione ed emancipazione. 1 Ed. Roma: Aracne Editrice, 2010.

CANADELLI, Elena (org.). Milano Scientifica 1875-1924, Volume 1: La rete del Grande Politecnico, 1 Ed. Milano: Sironi Editore, 2008.

CANADELLI, Elena; ZOCCHI Paola (orgs) Presentazione dellopera, in CANADELLI Elena (org.) Milano Scientifica 1875-1924, Volume 1: La rete del Grande Politecnico. 1 Ed. Milano: Sironi Editore, 2008.pp. 9-12.

CIVES, Giacomo; TRABALZINI Paola. Maria Montessori tra scienza spiritualità e azione sociale. 1 Ed. Roma: Anicia, 2017.

COVATO, Carmela. Un'identità divisa. Diventare maestra in Italia, tra Otto e Novecento. 1 Ed. Roma: Archivio Guido Izzi, 1996.

CUNNINGHAM, Hugh. Children and childhood in Western society since 1500. $1 \mathrm{Ed}$. Longman: London and New York: 1995.

DE GIORGI, Fulvio (org.), Annali di storia dell'educazione e delle istituzioni scolastiche. Sezione monografica: Maria Montessori e le sue reti di relazioni, n. 25, Brescia giugno 2018 a

DE GIORGI, Fulvio. Maria Montessori tra modernisti, antimodernisti e gesuiti, Annali di storia dell'educazione e delle istituzioni scolastiche. Sezione monografica: Maria Montessori e le sue reti di relazioni, n. 25, Brescia giugno 2018b, p. 27-73.

DE SANCTIS, Luigi (org.). L'infanzia svantaggiata e Maria Montessori. Esperienze psicopedagogiche, educative e sociali dal '900 ad oggi. 1 Ed. Roma: Fefè Editore, 2013.

DI POL, Redi Sante. La scuola per tutti gli italiani. L'istruzione di base tra Stato e società dal primo Ottocento ad oggi. 1 Ed. Milano: Mondadori, 2016.

GALLERANI, Manuela. Maria Montessori: “Donna nuova” e intellettuale impegnata nella (ri) scoperta dell'infanzia. In CAGNOLATI, Antonella. Maternità militanti. Impegno sociale tra educazione ed emancipazione. 1 Ed. Roma: Aracne Editrice, 2010. p. 83-113.

LIVI, Paola. Il Museo civico di Storia naturale tra collezioni, didattica e ricerca sperimentale. In: CANADELLI, Elena (org.). Milano Scientifica 1875-1924, Volume 1: La rete del Grande Politecnico. 1 Ed. Milano: Sironi Editore, 2008. p. 119-138.

MACINAI, Emilano. L'infanzia e i suoi diritti. Sentieri utopici, scenari globali e emergenze educative. 1 Ed. Pisa: ETS Edizioni, 2006.

MAPELLI, Barbara; SEVESO, Gabriella. (orgs.) Una storia imprevista. Femminismi del Novecento ed educazione, 1 Ed. Milano: Guerini Editore, 2006.

MERLO, Giordana. La prima infanzia e la sua educazione tra utopia e scienza dall'Età moderna al Novecento. 1 Ed. Milano: FrancoAngeli, 2011. 
MONTESSORI, Maria. Miserie sociali e nuovi ritrovati della scienza. I1 risveglio educativo, Roma, n.17, p. 130-132, 1898.

MONTESSORI, Maria. Influenze delle condizioni di famiglia sul livello intellettuale degli scolari. Ricerche di igiene e antropologia pedagogiche in rapporto all'educazione. Rivista di filosofia e scienze affin,. Roma, n. 3-4, p. 278-322, 1904.

MONTESSORI, Maria. Discorso inaugurale in occasione dell'apertura di una Casa dei bambini nel 1907, in MONTESSORI, Maria. La scoperta del bambino. 1. Ed. Milano: Garzanti, 1999 a, p. 361-373.

MONTESSORI, Maria. La scoperta del bambino. 1 Ed. Milano: Garzanti, 1999b.

MONTESSORI, Maria. Come educare il potenziale umano. 1 Ed. Milano: Garzanti, 2007.

PIRONI, Tiziana. Da Ellen Key a Maria Montessori: la progettazione di nuovi spazi educativi per l'infanzia. Ricerche di Pedagogia e Didattica, Bologna, n. 5, p. 1- 15, 2010 a.

PIRONI, Tiziana. Femminismo ed educazione in età giolittiana. Conflitti e sfide della modernità.1 Ed. Pisa: ETS Edizioni, 2010b.

PIRONI, Tiziana. La progettazione di nuovi spazi educativi per l'infanzia: da Ellen Key a Maria Montessori, Studi sulla Formazione, Firenze, n. 1, p. 81-89, 2010c.

PIRONI, Tiziana. Percorsi di pedagogia al femminile. Dall'Unità d'Italia al secondo dopoguerra. 1 Ed. Roma: Carocci, 2010d.

PIRONI, Tiziana. Maria Montessori e la formazione degli insegnanti per una nuova scuola. METIS, Bari, n. 12, p. 40-45, 2017.

PIRONI, Tiziana. Maria Montessori e gli ambienti milanesi dell'Unione Femminile e della Società Umanitaria. Annali di storia dell'educazione e delle istituzioni scolastiche. Sezione monografica: Maria Montessori e le sue reti di relazioni, Brescia, n. 25, p. 8-26, 2018.

REGNI Raniero. Infanzia e società in Maria Montessori. 1 Ed. Roma: Armando Editore, 2007.

SCOCCHERA Augusto. Maria Montessori. Una storia del nostro tempo. 1 Ed. Roma: Edizioni Opera Nazionale Montessori, 2005.

SEVESO, Gabriella Il diritto delle bambine all'istruzione sulle pagine di due riviste dell'inizio del Novecento: 'Unione Femminile' e 'La difesa delle lavoratrici'. Diacronie. Studi di Storia Contemporanea, n. 34, giugno 2018. Disponível em: <http://www.studistorici.com/2016/12/29/ seveso_numero_34/ >. Acesso em: 30 agosto 2018.

TOGNON, Giuseppe. Polvere di stelle. Maria Montessori e i confini nella costruzione dell'uomo. In DE SANCTIS, Luigi (org.). L'infanzia svantaggiata e Maria Montessori. Esperienze psicopedagogiche, educative e sociali dal ' 900 ad oggi. 1 Ed. Roma: Fefè editore, 2016. p.5-23.

TORNAR, Clara. La pedagogia di Maria Montessori tra teoria e azione, 1 Ed. Milano: FrancoAngeli, 2007.

TRABALZINI, Paola. Maria Montessori da Il Metodo a la scoperta del bambino. $1 \mathrm{Ed}$. Roma: Aracne Editrice, 2003.

TRABALZINI, Paola. Maria Montessori insegnante. Vita dell'Infanzia, Roma, n. 11-12, p. 14-23, 2016.

TRABALZINI, Paola. Maria Montessori, giustizia e bisogni sociali. 1 Ed. Roma: Edizioni Opera Nazionale Montessori, 2018.

ZOCCHI, Paola (org.) Milano Scientifica 1875-1924. Volume 2: La rete del perfezionamento medico. 1 Ed. Milano: Sironi Editore, 2008. . 
Notas

${ }^{1}$ Come è noto, il testo in realtà è stato scritto dall'Autrice nel 1909 con il titolo Il metodo della Pedagogia Scientifica applicato all'Educazione infantile nelle Case dei Bambini; in seguito, ha subito numerosi rimaneggiamenti e riedizioni ed è poi stato pubblicato con un titolo differente, La scoperta del bambino. In questo contributo faremo riferimento all'edizione del 1999 di Garzanti che comprende il testo del 1948, rivisto dall'Autrice, la Prefazione e il Discorso inaugurale in occasione dell'apertura di una Casa dei bambini nel 1907: per una ricostruzione attenta e dettagliata, si veda TRABALZINI, 2003.

${ }^{2}$ Una ricostruzione accurata di questo complesso progetto mostra come ciò che accadeva nella Milano scientifica del passato riveli «temi ancora oggi di grande attualità, che invitano a riflettere sul ruolo dei privati nel finanziamento alla ricerca scientifica e all'assistenza sanitaria, sui compiti e sulle finalità dell'istruzione universitaria e postuniversitaria, sul ruolo stesso della classe dirigente nell'ideazione di politiche culturali innovative e di ampio respiro» (CANADELLI; ZOCCHI, 2008, p. 11).

${ }^{3}$ Sulla parentela vera o presunta e sulle ricadute riguardo alla formazione della Montessori, si veda DE GIORGI, 2018b, pp. 27-28.

${ }^{4}$ Un'indagine molto dettagliata della complessa rete di relazioni all'interno della quale si mosse Maria Montessori è condotta con originalità, estrema accuratezza e profonda sensibilità nel numero monografico 25/2018 della rivista Annali di storia dell'educazione e delle istituzioni scolastiche pubblicato recentemente a cura di Fulvio DE GIORGI, il quale sottolinea nella "Premessa" che l'indagine consente di «fugare definitivamente - con pura attenzione filologica e storica, senza alcuno spirito apologetico preconcetto - quella sorta di impressione di opportunismo che potrebbe talvolta nascere d una solo estrinseca e superficiale conoscenza di tali relazioni, stereotipatamente assunte, senza approfondirne criticamente le evoluzioni (e involuzioni e interruzioni e riprese), le precise cronologie, le interferenze diacroniche e sincroniche: aspetti che solo una ricostruzione storica, rigorosamente condotta su basi documentate, può lumeggiare» (DE GIORGI, 2018 a, p.5).

* Professora associada do Departamento de Ciências Humanas para a Formação "Riccardo Massa" da Università degli Studi di Milano-Bicocca, Milão, Itália.

\section{Correspondência}

Gabriella Seveso - Università degli Studi di Milano-Bicocca. U06, Piano: P04, Stanza: 4136. Piazza dell'Ateneo Nuovo, 1 - 20126. Milano, Itália.

E-mail: gabriella.seveso@unimib.it

Recebido em 02 de setembro de 2018

Aprovado em 10 de setembro de 2018

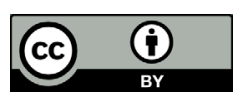

This work is licensed under a Creative Commons Attribution 4.0 International (CC BY 4.0). 